\title{
Ostéointégration et maladies générales. Recommandations cliniques
}

\section{RÉSUMÉ}

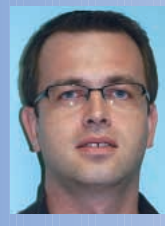

Sébastien GALLINA

Assistant hospitalo-universitaire, Département de parodontologie et d'implantologie orale, Faculté d'odontologie de Nancy,

Avenue de Lattre de Tassigny, 54000 Nancy.

Émilie REYES

Faculté d'odontologie de Nancy.

Catherine BISSON-BOUTELLIEZ

Maître de conférences,

Faculté d'odontologie de Nancy.

\section{Jean-Marc MARTRETTE}

Maître de conférences,

Faculté d'odontologie de Nancy.

\section{Neal MILLER}

Maître de conférences,

Faculté d'odontologie de Nancy.

Pascal AMBROSINI

Professeur des universités,

Faculté d'odontologie de Nancy.

L'ostéointégration dans un os sain est un processus largement documenté et maîtrisé. De nombreuses données montrent que ce processus peut être perturbé chez des patients souffrant de maladies générales ou polymédicamentés. Le but de cet article est de fournir au praticien des recommandations cliniques quant à leur influence sur l'ostéointégration des implants.

- implants

- ostéointégration

- maladies générales
AOS 2009;248:369-379

DOI: 10.1051/aos/2009411

C AEOS / EDP Sciences 


\section{Introduction}

La fiabilité des systèmes implantaires disponibles sur le marché et la prédictibilité des résultats permettent d'intégrer les implants au quotidien dans la réhabilitation prothétique des patients édentés. L'augmentation de l'espérance de vie et l'existence d'un quatrième âge conduisent à traiter de plus en plus de patients âgés. L'éventail de patients candidats à l'implantologie se trouve donc considérablement élargi. Pour toutes ces raisons, le clinicien implantologiste est plus fréquemment confronté à des patients souffrant de maladies systémiques ou polymédicamentés. La littérature scientifique montre qu'un certain nombre de maladies systémiques ou leurs traitements peuvent avoir une influence sur l'intégration osseuse des implants ainsi que sur sa pérennité. La santé des tissus mous péri-implantaires est également susceptible d'être perturbée par un certain nombre de désordres systémiques. L'implantologiste doit disposer d'éléments clairs sur ces pathologies avant toute thérapeutique.

\section{Les affections cardio-vasculaires}

Les études les plus récentes montrent que les patients atteints de maladies cardio-vasculaires ne présentent pas de risque augmenté d'échec de l'ostéointégration lorsque ces maladies sont traitées et contrôlées [1-3]. II n'existe pas de différence significative dans le taux d'échec implantaire entre des patients atteints d'HTA et des patients sains $[4,5]$.

\section{Les affections endocriniennes}

Le diabète (type I ou type II) peut être à I'origine de nombreuses complications systémiques parmi lesquelles des complications infectieuses et une altération de la guérison des plaies. Au niveau de la cavité buccale, il est souvent à l'origine d'une xérostomie, d'une augmentation du taux de glucose salivaire, ainsi que d'une augmentation de l'incidence des caries et des parodontites $[6,7]$. Le risque de parodontite est augmenté de 2,9 à 3,4 fois chez les patients diabétiques non ou mal équilibrés [8]. Ces patients restent en effet susceptibles à l'infection en raison du dysfonctionnement de leur système de défense. En effet, la chimiotaxie périphérique, la phagocytose ainsi que la destruction des bactéries par les polymorphonucléaires sont diminuées. La maladie microvasculaire est également susceptible d'augmenter la sensibilité à I'infection. Des études histomorphométriques montrent chez le patient diabétique une diminution de la formation et de la minéralisation osseuse [9-11]. La densité minérale osseuse chez un diabétique de type I est au moins $10 \%$ plus faible que celle d'un patient en bonne santé [1]. Le processus d'ostéointégration risque donc fort d'être perturbé chez le patient diabétique non contrôlé. II n'existe toutefois pas de preuve que cette maladie représente 
une contre-indication à l'implantologie chez le patient sous antidiabétiques oraux (diabète type II) ou sous insuline (type I) [11]. Plusieurs auteurs identifient l'ancienneté du diabète comme un facteur de risque supplémentaire qui augmenterait les complications microvasculaires classiques contribuant à l'échec, les rétinopathies et les néphropathies [12-13]. L'ancienneté serait en effet responsable d'une mortalité vasculaire plus importante $[3,11]$. Ainsi, seul le jugement clinique permettra au praticien de poser l'indication ou la contre-indication de la chirurgie. Un patient souffrant d'une maladie intervenue tardivement, suivant un régime alimentaire équilibré, et dont l'édentement n'est pas dû à une parodontite, présente un risque d'échec faible. À l'inverse, un patient insulinodépendant, chez qui la maladie parodontale est à l'origine de la perte de nombreuses dents, présente des risques d'échecs plus importants [14]. La chirurgie devra être réalisée de la manière la moins traumatisante possible sous couvert d'agents antimicrobiens (antibioprophylaxie pré- ou postopératoire, bains de bouche à la chlorhexidine), et sous une glycémie contrôlée [15].

Le traitement à l'insuline aurait peu d'impact sur l'ostéointégration.

\section{Insuffisance rénale chronique}

L'insuffisance rénale chronique est une contreindication absolue à la chirurgie implantaire. Les multiples complications de cette pathologie atteignent la quasi-totalité des systèmes (infection rénale récurrente, développement de tumeurs bénignes volumineuses ou malignes, diabète non contrôlé et/ou complications survenant de calculs rénaux). Le métabolisme et l'élimination des médicaments sont par ailleurs très perturbés. Les infections mal contrôlées aggravent la situation clinique des patients [16]. La destruction des néphrons peut conduire à une destruction osseuse par perte de calcium urinaire et par interruption de la production du métabolite actif vitamine $D$. Le manque de réabsorption calcique et le trouble fonctionnel de la parathormone conduisent à une ostéopénie et à une rétention d'endotoxines plasmatiques accompagnée de risques d'infections majeures. De ce fait et parce que l'évolution de la maladie est imprévisible, les implants dentaires sont strictement contreindiqués chez ces patients [17].

\section{Patients souffrant d'ostéoporose sous biphosphonates}

L'ostéoporose est une maladie systémique du squelette, caractérisée par une diminution de la masse osseuse. La détérioration de la microarchitecture trabéculaire conduit à une fragilité osseuse accrue ainsi qu'à une susceptibilité à la fracture $[3,18]$. Les modifications métaboliques liées à l'ostéoporose susceptibles d'interférer avec l'ostéointégration sont la diminution de la synthèse ou du métabolisme de la vitamine D, I'inhibition de l'absorption calcique, l'élévation du taux de parathormone et la diminution du taux de calcitonine et un 
remaniement osseux au cours duquel la résorption prédomine sur la formation [19]. Plusieurs études animales suggèrent un effet négatif de l'ostéoporose sur le pourcentage de contact os/implant et sur la densité osseuse péri-implantaire $[20,21]$. Les changements métaboliques et la perte osseuse visent préférentiellement l'os le plus actif métaboliquement, l'os spongieux [22, 23]. L'ostéoporose est donc un facteur de risques d'échecs surtout au maxillaire, principalement composé d'os spongieux [14, 15]. Toutefois, I'ostéointégration d'implants maxillaires et mandibulaires peut être obtenue même dans un os ostéoporotique après un délai plus important [24]. L'échec implantaire n'est pas corrélé à l'âge ou au sexe du patient. Il est associé à une qualité osseuse insuffisante [25]. Les caractéristiques biomécaniques de l'os ostéoporotique semblent similaires à celles de l'os de type IV qui a été associé à un taux de succès diminué [26]. Ces auteurs suggèrent la nécessité d'allonger le temps de cicatrisation. La diminution de la densité osseuse se traduit par une diminution de la résistance mécanique, des altérations de I'architecture osseuse, une teneur en minéraux diminuée et un ratio carbonate/phosphate plus élevé. L'os présente alors un risque augmenté de fracture ou de dégradation du lit implantaire. L'implantologiste devra en tenir compte lors de la chirurgie [24-27]. L'évaluation pré-implantaire de la qualité osseuse et de la trabéculation du futur site implantaire diminue les risques $[3,14]$.

L'ostéoporose ne constitue donc pas une contre-indication absolue aux implants. Un certain nombre d'études réalisées chez les patients atteints d'ostéoporose rapporte une ostéointégration réussie et des taux de succès implantaires très satisfaisants malgré la déperdition calcique $[3,28]$. La charge supportée par les implants endo-osseux stimule la formation osseuse mandibulaire [18, 25]. La fonction masticatoire recréée par les implants conduit à moyen terme à une densification de l'os périimplantaire [24]. Selon les recommandations de I'ANDEM (Agence nationale pour le développement et l'évaluation médicale), I'implantologie en milieu ostéoporotique ne représente qu'une contre-indication relative, qui cependant est un facteur favorisant de fractures mandibulaires après implantation. La mandibule est principalement constituée d'os cortical et les traitements contre l'ostéoporose ont tendance à augmenter la densité minérale osseuse (DMO). L'os devient plus cassant et possède une résistance élastique amoindrie face aux contraintes transmises par les implants.

L'ADA (Association dentaire américaine) préconise les recommandations suivantes pour le traitement implantaire des patients sous biphosphonates [29] :

- il est préférable de choisir des traitements alternatifs moins invasifs et non chirurgicaux. Les patients devront être informés des risques en cas de thérapeutique implantaire ; - une régénération osseuse guidée (ROG) et la pose de plusieurs implants augmentent les risques. Lorsque la chirurgie implique plusieurs sextants, il est préconisé de traiter un sextant à la fois avec une interruption de 2 mois entre chaque intervention :

- la chirurgie doit être effectuée par un praticien expérimenté. La technique utilisée doit être atraumatique et la fermeture des tissus mous hermétique ;

- une antibiothérapie systémique et un rinçage pré- et postopératoire à la chlorhexidine sont recommandés ;

- pour un patient sous biphosphonate oral depuis moins de 3 ans, il n'existe pas de risque clinique. Le contrôle clinique et radiologique minimise les risques potentiels. Le patient sera placé sous surveillance et rappels réguliers. II 
devra être informé des risques d'échecs implantaires et d'ostéonécrose en cas de poursuite du traitement. Le praticien prendra contact avec le médecin prescripteur et suggérera une modification de la dose administrée ou le choix d'une autre médication ;

- pour un patient sous biphosphonates et stéroïdes pendant moins de 3 ans, ou sous biphosphonates, avec ou sans stéroïdes (exemple: prednisone), pendant plus de 3 ans, le traitement devra être suspendu au moins 3 mois avant la chirurgie à condition que l'état général du patient le permette. Le médicament ne peut être repris avant que la cicatrisation osseuse ne soit complète.

Un certain nombre d'études montre que l'administration d'ibandronate après la chirurgie implantaire peut améliorer la stabilité implantaire, l'ostéointégration, et probablement le taux de survie implantaire à long terme $[30,31]$.
D'autres études rapportent que l'alendronate est un traitement efficace dans la prévention de perte de masse osseuse autour des implants intégrés [32, 33]. Des évaluations biomécaniques montrent que l'alendronate augmenterait le torque nécessaire pour dévisser les implants [34]. L'alendronate oral serait donc favorable à l'ostéointégration par son effet anabolique [35]. II préviendrait l'effet négatif de la déficience œstrogénique sur l'ostéointégration. Son effet serait prolongé après l'arrêt d'administration contrairement à l'œstradiol [36].

L'Agence française de sécurité sanitaire des produits de santé (AFSSAPS) contre-indique l'implantologie chez des patients traités par biphosphonate IV. Aucune recommandation n'a été établie pour les biphosphonates oraux. L'implantologie est contre-indiquée chez le patient sous biphosphonates depuis plus de 3 ans [37].

\section{Patient souffrant d'ostéomalacies}

L'ostéomalacie est une maladie rare de l'adulte et du sujet âgé caractérisée par une déminéralisation généralisée du tissu osseux. Elle est due à une insuffisance de fixation du calcium et du phosphore [3, 38]. Aucune preuve n'existe quant à l'influence de l'ostéomalacie sur l'intégration des implants. Un certain nombre de patients souffrant de cette maladie ont pu être auparavant rattachés aux groupes de patients ostéoporotiques qui présentent un os de faible

\section{Affections malignes}

Le cancer en évolution et/ou en traitement (hémopathies malignes, cancers des voies aéro-digestives) contre-indique la chirurgie implantaire. Les complications infectieuses perturbent l'obtention de l'ostéointégration qualité [3]. II n'existe aucune contre-indication à la chirurgie implantaire chez des patients médicalement contrôlés. Le seul risque encouru est la non-ostéointégration du ou des implants [17]. Certains patients souffrant d'une ostéomalacie résistent à leur traitement. Ils sont souvent atteints de la maladie de Rickets. La chirurgie implantaire chez de tels patients est contre-indiquée en raison d'un risque infectieux augmenté et de la non-ostéintégration des implants [16].

et aggravent le pronostic vital du patient. Le risque infectieux est majeur pour des taux de lymphocytes T CD4+ inférieurs à 200 cellules $/ \mathrm{mm}^{3}$. Karr et al. rapportent des complications (infection avec perte osseuse, mobilité 
implantaire, maladie péri-implantaire chronique, hémorragie et mucosite) apparues après une chimiothérapie administrée à la suite d'une chirurgie implantaire [39]. L'indication peut être réévaluée en concertation étroite avec l'équipe médicale pour les patients guéris (souvent après 5 ans de rémission complète, sans récidive ni deuxième localisation, ni deuxième cancer) [17]. La chirurgie implantaire est contre-indiquée au cours de la phase active d'une chimiothérapie ainsi que lors des six mois suivants l'administration de plusieurs agents anticancéreux [16].

La chimiothérapie (méthotrexate, raltitrexed...) peut affecter la cicatrisation osseuse. Les muqueuses orales sont fréquemment affectées par des stomatites, des mucosites et des gingivites péri-implantaires. Ces altérations des tissus mous sont susceptibles de compromettre l'intégration des implants. Les effets secondaires tardifs tels qu'une altération des cellules vasculaires conduisent également à une malnutrition de l'os. Cela peut induire une perte osseuse similaire à celle de l'ostéoporose, responsable de taux de survie implantaire diminués. Kovacs rapporte un remodelage osseux diminué, spécialement à l'interface os/implant, lors de chimiothérapie utilisant le cisplatine [40]. II suggère le recouvrement muqueux des implants lors de la période cicatrisation (3 mois). Cette précaution les protège d'une infection éventuelle qui peut compromettre l'ostéointégration.

\section{Sida et ostéointégration}

L'immunodépression induite par le VIH et la neutropénie sont des éléments importants dans la survenue de complications postopératoires précoces et tardives (septicémie, cicatrisation osseuse retardée). Des infections opportunistes initiales (candidoses orales) apparaissent lorsque les valeurs de lymphocytes $T$ helper (CD4+) sont inférieures à 400 cellules $/ \mathrm{mm}^{3}$ [41]. Lorsque le nombre de CD4+ est inférieur à 200 cellules $/ \mathrm{mm}^{3}$, les patients sont sujets aux infections majeures en raison du déficit immunitaire. Harrison et al. montrent une augmentation de l'incidence des infections de $42 \%$ chez le patient HIV positif, et de
$11 \%$ chez le patient HIV négatif lorsqu'il se produit une contamination préopératoire [42]. Toutefois, Achong et al. concluent qu'un faible taux de CD4+ $(<200 / \mu \mathrm{L})$ ne prédit pas une susceptibilité augmentée aux infections de lésions intra-orales, ou à des taux d'échecs implantaires élevés [43]. La pose d'implants n'est donc pas contre-indiquée chez les patients séropositifs sous trithérapies et asymptomatiques. Le contrôle des lymphocytes T4 doit révéler des taux supérieurs à 200/ $\mathrm{mm}^{3}$ (le taux normal de $\mathrm{CD} 4+$ étant de 1000 à $1500 / \mathrm{mm}^{3}$ ). Le dosage de l'antigène P24 doit être négatif $[27,44]$.

\section{Maladies systémiques auto-immunes}

Le syndrome de Papillon-Lefèvre ou hyperkératose palmo-plantaire est une maladie rare de la peau due à des mutations (mode autosomique récessif) dans le gène cathepsine $C$ qui affec- tent la réponse immune innée [45]. Plusieurs auteurs rapportent des cas d'implantations réussies et aucun rapport ne mentionne de contre-indication implantaire [46, 47]. 
Toutefois le manque de données cliniques rend l'indication implantaire chez de tels patients extrêmement imprédictible. Des précautions sont requises vis-à-vis de l'âge physiologique du patient et de son état de santé générale [45].

Le lupus érythémateux systémique est une maladie auto-immune qui affecte plusieurs systèmes d'organes. Les articulations, les reins, le cœur et les poumons sont les plus fréquemment touchés. Ces patients présentent une susceptibilité accrue aux infections opportunistes.

La sclérodermie également appelée sclérose systémique progressive est une maladie autoimmune chronique. Elle touche de préférence la peau (aspect brillant et tendu), le tractus gastro-intestinal, les poumons, le cœur, les reins et le système musculo-articulaire. La fibrose tissulaire généralisée est due à l'augmentation de la synthèse du collagène de type I et III au niveau cutané et viscéral (sclérose collagène). La sclérodermie se caractérise également par une dysfonction endothéliale des petits vaisseaux qui se traduit par des lésions inflammatoires et vasculaires (vasospasmes, fibrose de l'adventice), et par la formation d'auto-anticorps dirigés contre un certain nombre de composants tissulaires [25]. Très peu d'informations sont disponibles sur l'impact de la sclérodermie sur l'ostéointégration. Quelques rapports évoquent une réhabilitation orale à l'aide d'implants ostéointégrés réussie $[48,49]$.

\section{La trisomie 21 ou syndrome de Down}

C'est la plus fréquente des anomalies chromosomiques (prévalence de 1/700 naissances). Ce désordre génétique conduit souvent à une perte dentaire en raison d'une augmentation de la susceptibilité aux infections. On pourrait penser que la susceptibilité aux infections parodontales augmente aussi le risque d'échec implantaire. Une coopération rigoureuse et suivie dans le temps est primordiale. Bien que d'autres études soient nécessaires, le recours à I'implantologie chez ces patients paraît envisageable $[49,50]$.

\section{Patients sous médicaments antirejets}

Les dihydropyridines et la cyclosporine ont pour effet commun une hypertrophie gingivale non plaque-dépendante [3]. Cet effet secondaire entrave I'hygiène orale et les soins de maintenance professionnelle. Il existe donc un risque accru de péri-implantite qui peut compromettre l'obtention et le maintien de l'ostéointégration. Les données bibliographiques qui rapportent l'impact des médications antirejets sur l'intégration des implants sont peu nombreuses. Le pronostic semble défavorable pour la cyclosporine. Elle entraîne en effet une ostéoporose, une accélération du remodelage osseux et une balance osseuse négative [3]. Le statut immun du patient est également affaibli [43]. Un certain nombre d'études animales rapportent un effet négatif de la cyclosporine A et de la nifédipine sur l'ostéointégration [51]. Sakakura et al. montrent que la cyclosporine A diminue la force nécessaire pour dévisser des implants ostéointégrés et le pourcentage de contact os/implant [52]. La nifédipine réduit la surface osseuse périimplantaire [51]. 


\section{Conclusion}

Les preuves scientifiques apportées par la littérature montrent qu'il existe un certain nombre de maladies systémiques ou de traitements qui influencent le processus d'intégration osseuse des implants en modifiant le métabolisme du patient. En effet, la réponse osseuse à l'implantation diffère selon les conditions métaboliques systémiques et les conditions morphologiques du site implantaire. Un certain nombre de maladies ou la prise de certains médicaments sont des contre-indications absolues à l'implantologie. Ce type de situation reste somme toute relativement rare. Dans de nombreux cas, le recours à l'implantologie reste possible même si les maladies en cause ou les traitements repré- sentent un facteur de risque d'échec précoce ou tardif de l'ostéointégration. Le respect d'un certain nombre de précautions permet toutefois de limiter les risques d'échecs et de complications postopératoires. Une prise en charge individuelle et une collaboration étroite avec l'équipe médicale qui suit le patient permettent de prendre en charge le patient de la manière la plus globale. Le bilan biologique pré-implantaire affine la sélection du patient et optimise les chances de succès. Chez ce type de patients, le maintien d'une hygiène orale satisfaisante et le rétablissement d'une fonction masticatoire font partie intégrante du plan de traitement global de la maladie.

\section{Bibliographie}

1. Elsuheibi ES, Zarb GA. Implant prosthodontics in medically challenged patients: the university of Toronto experience. J Can Dent Assoc 2002;68(2):103-108

2. Khavidi V, Anderson J, Zarb GA.

Cardiovascular disease and treatment outcomes with osseointegration surgery.

J Prosthet Dent 1999;81(5):533-536.

3. Van Steenberghe $D$, Quirynen M, Molly L et al. Impact of systemic diseases and medication on osseointegration.
Periodontol 2000 2003:33:163-171.

4. Alssadi G, Quirynen M Komarek A et al. Impact of local and systemic factors on the incidence of oral implant failures, up to abutment connection. J Clin Periodontol 2007:34(7):610-617.

5. Alssadi G, Quirynen M, Michiles $\mathrm{K}$ et al. Impact of local and systemic factors on the incidence of failures up to abutment connection with modified surface oral implants.
J Clin Periodontol 2008;35(1):51-57.

6. Murrah A

Diabetes mellitus and associated oral manifestations: a review. J Oral Pathol 1985;14(4):271-81.

7. Reest $D$. Periodontal management of the patient with diabetes mellitus. Periodontol 2000;23:63-72.

8. Klokkevold P R. Periodontal medicine: assessment of risk factors for disease.

J Can Dent Assoc 1999;27(2):135-42.

9. Kopman JA, Kim DM, Rahman SS et al. 
Modulating the effects of diabetes on osseointegration with aminoguanadine and doxycycline.

J Periodontol 2005;76(4):614-20.

10. Takeshita F, lyama $S$, Ayukawa $Y$ et al. The effects of diabetes on the interface between hydroxyapatite implants and bone in rats tibia.

J Periodontol 1997;8(2):180-185.

11. Giannobile WV, Rios HF, Lang NP.

Bone as a tissue.

In: Clinical periondontology and implant dentistry.

Oxford: Blackwell

Munksgaard

2008:XX-1340-i26p.

12. Olson JW, Shernoff AF, Tarlow JL et al.

Dental endosseous implant assessment in a type 2 diabetic population:

a retrospective study. J Oral Maxillofac Implants 2000;15(6):811-818.

13. Peled M, Ardekian L, Tagger-Green $\mathrm{N}$ et al. Dental implants in patients with type 2 diabetes mellitus: a clinical study. Implant Dent 2003;12(2):116-22.

14. Bugea $C$, Luongo $R$, Di Lorio $\mathrm{D}$ et al. Contact os-implants ostéo-intégrés : analyse histologique d'un implant à la surface mordancée chez un patient diabétique. Parod Dent Restaur 2008;28:145-151.
15. Paquette DW, Bordala N, Williams RC.

Risks factors

for endosseous dental implant failure.

Dent Clin North Am 2006;50(3):361-374.

16. Chavanaz M.

Patient screening and medical evaluation for implant and presprosthetic surgery. J Oral Implantol 1998;24(4):222-229.

17. Kleinfinger $S$, Goldberg $M$, Khayat $\mathrm{P}$ et al. Implantologie orale. Paris : Association dentaire française 2003:143 p.

18. Bianchi A, Sanfilipo F. L'ostéoporose : effet sur la résorption de l'os maxillaire et options thérapeutiques implantaires.

Parod Dent Restaur 2002;22:231-239.

19. Roberts WE, Simmons $K E$, Garetto LP et al. Bone physiology and metabolism in dental implantology: risk factors for osteoporosis and other metabolic bone diseases.

Implant Dent 1992;1(1):11-21.

20. Cho P, Scheinder GB, Krigan $\mathrm{K}$.

Examination of the bone interface in experimentally induced osteoporotic bone. Implant Dent 2000;13(1):79-87.

21. Sakakura $C E$, Giro $G$, Goncalves D et al. Radiographic assessment of bone density around integrated titanium implants after ovariectomy in rats.
Clin Oral Implants Res 2006;17:134-138.

22. Duarte PM, Cesar JB, Goncalves PF et al. Estrogen deficiency affects bone healing around titanium implants: a histometric study in rats. Implant Dent 2003;12(4):340-6.

23. Pan J, Shirota T, Ohno K et al. Effect of ovariectomy on bone remodeling adjacent to hydroxyapatite coated implants in the tibiae of mature rats. J Oral Maxillofac Surg 2000;58(8):877-882.

24. Sanfilipo F, Bianchi AE. L'ostéoporose : effet sur la résorption de l'os maxillaire et options thérapeutiques implantaires : revue de la littérature et considérations cliniques. Parod Dent Restaur 2003;23:447-57.

25. Sugerman PB, Barber MT. Patient selection for endosseous dental implants: oral and systemic considerations. J Oral Maxillofac Surg 2002;17(2):191-201.

26. Herrmann J, Lekholm U, Holm S et al. Evaluation of patient and implant characteristics as potential prognostic factors for oral implant failures.

Int J Oral Maxillofac Implants 2005;20(2):220-30.

27. Hwang D, Wang H-L. Medical contraindications to implant therapy: part II: relative contraindications. Implant Dent 2007;16(1):13-23. 
28. Friberg B, Ekestubbe A, Mellstrom D et al. Bränemark implants and osteoporosis: a clinical exploratory study. Clin Implant Dent Relat Res 2001;3(1): 50-66.

29. Grant B-T, Amenedo C, Freeman K et al. Outcomes of placing dental implants in patients taking oral biphosphonates: a review of 115 cases. J Oral Maxillofac Surg 2008;66(2):223-30.

30. Eberhardt C, Schwarz M, Brahnkamp J et al. lbandronate improves osseointegration of cementless metal implants to a similar extent either given continously. Bone 2006;38:548.

31. Kurth $A H$, Eberhardt $C$, Müller $S$ et al. The bisphosphonate ibandronate improves implant integration in osteopenic ovariectomized rats. Bone 2005;37(2):204-210.

32. Frenkel SR, Jaffe WL, Valle CD et al.

The effect of alendroante (fosamax) and implant surface on bone integration and remodeling in a canine model. J Biomed Mater Rest 2001;58(6):645-650.

33. Giro G, Goncalves D, Sakakura CE et al. Influence of estrogen deficiency and its treatment with alendronate and estrogen on bone density around osseointegrated implants: radiographic study in female rats.

Oral Surg Oral Med

Oral Pathol Oral Radiol

Endod 2008;105(2):162-7.

34. Giro G, Sakakura CE, Goncalves D et al. Effect of $17 \beta$ œstradiol and alendronate on the removal torque of osseointegrated titanium implants in ovariectomized rats. J Periodontol 2007;78(7):1316-1321.

35. Wang HL, Weber $D$, Mc Cauley LK. Effect of long-term oral bisphosphonates on implant wound healing: literature review and a case report. J Periodontol 2007;78(3):584-94.

36. Duarte PM, de Vasconcelos Gurgel BC, Sallum AW et al.

Alendronate therapy may be effective in the prevention of bone loss around titanium implants inserted in estrogen-deficient rats. J Periodontol 2005;76(1):107-114.

37. Scully C, Madrid C, Bagan J. Dental endosseous implants in patients on biphosphonate therapy. Implant Dent 2006;15(3):212-218.

38. Addad $\mathrm{H}$, Mas R. Biochimie Clinique. Paris : Vernazobres-Grego 2007; 1vol:280p.

39. Karr RA, Kramer DC, Toth BB.

Dental implants and chemotherapy complications. J Prosthet Dent 1992;67(5):683-687.
40. Kovacs AF.

Influence of chemotherapy on endosteal implant survival and success in oral cancer patients. Int J Oral Maxillofac Surg 2001;30(2):144-7

41. Etienne D, Sanz M, Aroca $S$ et al. Identification des patients à risque en implantologie orale (II). J Parodontol Implantol Orale 1998;17(3)98:273-297.

42. Harrison WJ, Lewis $C P$, Lavy CB.

Wound healing after implant surgery in HIV-positive patients. $\mathrm{J}$ Bone Joint Surg Br 2002;84(6): 802-806.

43. Achong RM, Shetty K, Arribas A et al. Implants in HIV-positive patients: 3 case reports. J Oral Maxillofac Surg 2006;64(8):1199-1203.

44. Ferreira SD, Silva GL, Cortelli JR et al. Prevalence and risk variables for peri-implant disease in Brazilian subjects. J Clin Periodontol 2006;33(12):929-935.

45. Beikler T, Flemming TF. Implants in the medically compromised patient. Crit Rev Oral Biol Med 2003;14(4):305-316.

46. Ulbro C, Crossner CG, Lundgren $\mathrm{T}$ et al. Osseointegrated implants in a patient with Papillon-Lefèvre Syndrome.

A 4 1/2 year follow-up. J Clin Periodontol 2000;27(12):951-954.

47. Woo I, Brunner DP, Yamashita DR et al. 
Dental implants

in a young patient with Papillon-Lefevre

Syndrome:

a case report.

Implant Dent

2003;12(2):140-144.

48. Hodgson TA, Lewis $\mathrm{N}$, Darbar U et al.

The short-term efficacy of osseointegrated implants in patients with non-malignant oral mucosal disease: a case series.

Oral Diseases 2006;12(s1):11.

49. Oczakir C, Balmer S, Mericske-Stern R.
Implant-prosthodontic

treatment

for special care patients:

a case series study.

Int J Prosthodont

2005; 18(5):383-389.

50. Lustig JP, Yanko R, Ziberman U.

Use of dental implants

in patients with

Down syndrome:

a case report.

Spec Care Dentist 2002;22(5):201-204.

51. Duarte PM,

Noqueira Filho GR,

Sallun EA et al.

The effect of an immunosuppressive therapy and its with drawal on bone healing

around titanium implants:

a histomorphometric study in rabbits.

J Periodontol

200;72(10):1391-7.

52. Sakakura CE, Margonar R, Holzhausen $\mathrm{M}$ et al. Influence of cyclosporine. A therapy on bone healing around titanium implants: a histomorphometric and biomechanic study in rabbits.

J Periodontol 2003;74(7):976-981.

\section{SUMMARY}

\section{Osteointegration and systemic diseases.}

\section{Clinical rationale}

Sébastien GALLINA,

Émilie REYES,

Catherine BISSON-BOUTELLIEZ, Jean-Marc MARTRETTE,

Neal MILLER,

Pascal AMBROSINI

$$
\begin{aligned}
& \text { Keywords } \\
& \text { - implants } \\
& \text { - osteointegration } \\
& \text { - systemic diseases }
\end{aligned}
$$

Osteointegration in a healthy bone site is a well documented and controlled biological process. Abundant data demonstrate difficulties in patients with systemic diseases or polymedicated. The goal of this article is to provide the practitioner with clinical rationale to obtain osteointegration. 
France et Union Européenne

version papier + version électronique $204 €$

Étudiant* : France et Union Européenne

(* sur présentation d'une attestation)

version papier + version électronique

Reste du monde

version papier + version électronique

Membre de I'AEOS

Prix au numéro

Frais d'envoi par avion

(*) Offre valable jusqu'au 31/10/2010

\section{NUMÉROS(*)}

TTC

$179 €$
$124 €$

$234 €$ $183 €$

$67 €$

$49 €$

$109 €$ version électronique seule

$149 € \square$

version électronique seule

Frais de port au numéro

$4 €$

Je souhaite m'abonner à la Revue AOS

Je souhaite prolonger mon abonnement à la Revue AOS

\section{MODE DE PAIEMENT :}

$\square$ Paiement effectué par chèque à l'ordre de : EDP Sciences - REVUE AOS

17 avenue du Hoggar - P.A. de Courtabœuf

B.P. 112 - 91944 Les Ulis Cedex A (France)

Tél. + 33 (0)169181514 - Fax + 33 (0)169860678

e-mail : abonnements@edpsciences.org

Paiement en ligne disponible sur notre site www.aos-journal.org
$\square$ Paiement par carte bancaire : $\square$ Carte Visa
$\square$ Master Card
American Express

$\mathrm{N}^{\circ}$

Noter ici les 3 derniers chiffres situés au dos de la carte

Date d'expiration

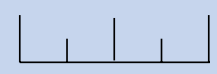

Signature obligatoire

VOTRE ADRESSE :

Vous êtes: $\square$ Chirurgien-Dentiste $\square$ Stomatologue $\square$ Orthodontiste $\square$ Autre:précisez

Nom :

Prénom :

Adresse :

Code Postal Ville

Tél

Fax E-mail. 